\title{
Role of calcineurin in Porphyromonas gingivalis-induced myocardial cell hypertrophy and apoptosis
}

\author{
Shin-Da Lee ${ }^{1}$, Wei-Wen $\mathrm{Kuo}^{2}$, Ding-Yu Lin ${ }^{3}$, Ter-Hsin Chen ${ }^{4}$, Wu-Hsien Kuo ${ }^{5}$, \\ Hsi-Hsien Hsu ${ }^{6}$, Jian-Zhi Chen ${ }^{7}$, Jer-Yuh Liu ${ }^{3}$, Yu-Lan Yeh ${ }^{8}$ \& Chih-Yang Huang ${ }^{3} *$ \\ ${ }^{1}$ School of Physical Therapy, Chung-Shan Medical University, Taichung, Taiwan; ${ }^{2}$ Department of Biological \\ Science and Technology, China Medical College, Taichung, Taiwan; ${ }^{3}$ Institute of Biochemistry, Chung-Shan \\ Medical University, No. 110, Section 1, Chien Kuo N. Rd., Taichung 40203, Taiwan, R.O.C; ${ }^{4}$ Graduate \\ Institute of Veterinary Public Health, National Chung-Hsing University, Taichung, Taiwan; ${ }^{5}$ Division of \\ Gastroenterology, Department of Internal Medicine, Armed-Force, Taichung General Hospital, Taichung, \\ Taiwan; ${ }^{6}$ Division of colorectal surgery, Mackay Memorial Hospital, Taipei, Taiwan; ${ }^{7}$ Division of Cardiol- \\ ogy, Show-Chwan Memorial Hospital, Changhua, Taiwan; ${ }^{8}$ Department of Pathology, Changhua Christian \\ Hospital, Changhua, Taiwan
}

Received 17 July 2005; accepted 15 November 2005

(C) 2005 National Science Council, Taipei

Key words: periodontitis, Porphyromonas gingivalis, myocardial cell, apoptotic, cell death, calcineurin signal pathways

\section{Summary}

Background and objective: Periodontal pathogen Porphyromonas gingivalis ( $P$. gingivalis) increased cardiomyocyte hypertrophy and apoptosis whereas Actinobaeillus actinomycetemcomitans and Prevotella intermedia had no effects. The purpose of this study is to clarify the role of calcineurin signaling pathway in P. gingivalis-induced H9c2 myocardial cell hypertrophy and apoptosis. Methods: DNA fragmentation, nuclear condensation, cellular morphology, calcineurin protein, Bcl2-associated death promoter (Bad) and nuclear factor of activated T cell (NFAT)-3 protein products in cultured H9c2 myocardial cell were measured by agarose gel electrophoresis, DAPI, immunofluorescence, and Western blotting following $P$. gingivalis and/or pre-administration of CsA (calcineurin inhibitors cyclosporin A). Results: P. gingivalis not only increased calcineurin protein, NFAT-3 protein products and cellular hypertrophy, but also increased DNA fragmentation, nuclear condensation and Bad protein products in $\mathrm{H} 9 \mathrm{c} 2$ cells. The increased cellular sizes, DNA fragmentation, nuclear condensation, and Bad of $\mathrm{H} 9 \mathrm{c} 2$ cells treated with $P$. gingivalis were all significantly reduced after pre-administration of CsA. Conclusion: Our findings suggest that the activity of calcineurin signal pathway may be initiated by $P$. gingivalis and further lead to cell hypertrophy and death in culture $\mathrm{H} 9 \mathrm{c} 2$ myocardial cells.

\section{Introduction}

The accumulation of epidemiologic, in vitro, animal and clinical evidence suggests that periodontal infection may be a contributing risk factor for

\footnotetext{
*To whom correspondence should be addressed. Phone: + 8864-24730022 ext. 11682; Fax: + 886-4-23248195; E-mail: chuang1@ csmu.edu.tw
}

heart diseases [1]. Periodontitis pathogens may disseminate through the blood to infect the vascular endothelium and contribute to the occurrence of atherosclerosis and risk of myocardial ischemia and infarction [2]. Porphyromonas $(P$. gingivalis, a major pathogen of chronic adult periodontitis, exhibits several potential virulence properties which could play a role in the development of cardiovascular disease as mediators of low 
density lipoprotein oxidation, foam cell formation, and rupture of atherosclerotic plaque [3]. The balance between cell death and cell survival is a tightly controlled process, especially in terminally differentiated cells, such as the cardiomyocyte [4]. Therefore, it becomes a very important issue whether $P$. gingivalis will induce cardiomyocyte apoptosis and hypertrophy. In our previous studies, $P$. gingivalis increased cellular sizes, apoptosis, and the activated apoptotic caspase-3, caspase-8, and caspase-9 proteins of cardiomyocyte $\mathrm{H} 9 \mathrm{c} 2$ cells whereas Actinobaeillus actinomycetemcomitans and Prevotella intermedia had no effects. [5]. Besides, $P$. gingivalis was demonstrated to adhere and to invade aortic and heart endothelial cells [6]. However, the role of calcineurin myocardial hypertrophy and apoptosis induced by $P$. gingivalis is still unclear.

Cardiac hypertrophy may initially be a beneficial adaptation that normalizes wall stress, but the prolonged cardiac hypertrophy is a leading cause of sudden death and heart failure [7]. Apoptosis had been reported to contribute to the loss of myocardial cells in cardiomyopathy, and is recognized as a predictor of adverse outcomes in subjects with cardiac diseases or heart failure [8]. Cardiac myocyte apoptosis may occur in a variety of diseases, such as hypertension, myocardial infarction, endocrine disorders, toxicants, and bacterial endocarditis [9, 10]. Apoptosis is characterized by DNAfragmentation, nuclear condensation, and shifting the balance between anti-apoptotic and pro-apoptotic proteins towards the latter. [11]. Members of the $\mathrm{Bcl} 2$ family are important regulators of programmed cell death and individual members that can suppress (e.g. Bcl2) or promote (e.g. Bad) apoptosis. The Bcl2-associated death promoter (Bad), a pro-apoptotic member of the Bcl-2 family, modulate death signaling and lead to release of cytochrome $c$ from the mitochondria into the cytoplasm, which results in DNA fragmentation and cell death by activation of caspase 9 and 3 [12].

Calcineurin is a calmodulin-dependent, serine/ threonine-specific phosphatase function within a signal transduction pathway that regulates gene expression and biological responses in many important cell types [13]. Calcineurin was considered as an essential mediator of cardiac hypertrophy and apoptosis, and in most studies, calcineurin inhibitors attenuated cardiac hypertrophy in vivo and also attenuated apoptosis of cardiac myocytes
[14]. Calcineurin plays a critical role in the apoptosis of cardiac myocytes through dephosphorylating Bad, thus enhancing Bad heterodimerization with Bcl-xL or other Bcl-2 family and promoting apoptosis. [14, 15]. Calcineurin transmits signals to the nucleus through the dephosphorylation and translocation of nuclear factor of activated $\mathrm{T}$ cell (NFAT) transcription factors. [13, 16]. Calcineurin and its direct downstream transcriptional effector NFAT-3 potentially play a regulatory role for maladaptive hypertrophy and heart failure [16]. In human heart failure, the calcineurin signaling pathway was activated not only by an increased activity of calcineurin but also by the shift from phosphorylated NFAT-3 to the nucleus dephosphorylated NFAT-3 [17]. Once activated, calcineurin will dephosphorylate NFAT3 and $\mathrm{Bad}$, allowing translocation of these proteins to the nucleus and mitochondria, respectively, both of which may potentially lead to myocardial pathologic hypertrophy and apoptosis [15]. However, it is unclear whether calcineurin signaling pathways are involved in $P$. gingivalis-associated myocardial cell hypertrophy and apoptosis.

The purpose of the current study is, first to determine the effect of periodontal pathogen $P$. gingivalis on calcineurin, Bad, and NFAT-3 protein levels, second to clarify whether $P$. gingivalis-induced $\mathrm{H} 9 \mathrm{c} 2$ myocardial cell hypertrophy is mediated via calcineurin signaling pathway, and third to evaluate whether calcineurin/Bad signaling pathways is related to $P$. gingivalis-induced $\mathrm{H} 9 \mathrm{c} 2$ myocardial cell apoptosis. We hypothesized that calcineurin activity may be related to Porphyromonas gingivalis-induced myocardial cell hypertrophy and apoptosis.

\section{Methods}

Porphyromonas gingivalis preparation

Porphyromonas (P.) gingivalis obtained from American Type Culture Collection (ATCC 33277) was maintained on brain heart infusion (BHI) enriched with Vitamin K3 (menadione, $0.5 \mathrm{mg} / \mathrm{l})$ and hemin $(5 \mathrm{mg} / \mathrm{l})$ in an atmosphere of $5 \% \mathrm{CO}_{2}, 10 \% \quad \mathrm{H}_{2}$, and $85 \% \quad \mathrm{~N}_{2}$ at $37{ }^{\circ} \mathrm{C}$. Turbidimetric method was used to evaluate bacterial cultures with 2 McFarland standard $\left(6 \times 10^{8}\right.$ colony forming units (CFUs)), the upper aqueous 
layer was collected following centrifugation at $10,000 \mathrm{~g}$, filtered through $0.22 \mu \mathrm{m}$ filter, and stored at $-80{ }^{\circ} \mathrm{C}$. The upper aqueous layers of $P$. gingivalis bacterial mediums were diluted to $10-$ fold and 100-fold.

\section{Cell culture}

H9c2 cardiac cells were obtained from American Type Culture Collection (ATCC CRL-1446) and were cultured in Dulbecco's modified essential medium supplemented with $10 \%$ fetal bovine serum, $2 \mathrm{mM}$ glutamine, 100 units $/ \mathrm{ml}$ penicillin, $100 \mu \mathrm{g} / \mathrm{ml}$ streptomycin, and $1 \mathrm{mM}$ pyruvate in humidified air $\left(\mathrm{CO}_{2} 5 \%\right)$ at $37{ }^{\circ} \mathrm{C}$. $\mathrm{H} 9 \mathrm{c} 2$ cells were cultured in serum-free medium for $12 \mathrm{~h}$, and then treated with control (serum-free medium with minimal essential medium) or CsA $(0.5 \mu \mathrm{M} / \mathrm{ml}$; calcineurin inhibitors cyclosporin A) for $1 \mathrm{~h}$ and post-treated with $P$. gingivalis bacterial 10-fold, or 100 -fold diluted bacterial mediums. The incubation was continued for another $0.5,1,2$ or $24 \mathrm{~h}$, and then the cells were harvested and extracted for the analysis.

\section{Actin-immunofluorescence (rhodamine-phalloidin)}

H9c2 cells were fixed in fresh $3.7 \%$ paraformaldehyde and permeabilized with $2 \%$ Triton X-100 for $10 \mathrm{~min}$, and blocked with PBS buffer with $1 \%$ bovine serum albumin (PBS) for $15 \mathrm{~min}$. Actin filaments were stained by rhodamine-phalloidin. Cells were examined and photographed using a Zesis Axioskop and a confocal microscope. The cell sizes were magnified 100 times and analyzed by digital image analysis, using Image-Pro Plus software.

\section{DNA fragmentation}

H9c2 cells were lysed in $50 \mu$ lof lysis buffer (50 mM Tris- $\mathrm{HCl}$ (pH 7.4), $20 \mathrm{mM}$ EDTA, $1 \%$ IGEPAL-630) followed by incubation with $1 \%$ SDS and $5 \mu \mathrm{g} / \mu \mathrm{l}$ RNase (Roche Molecular Biochemicals, Mannheim, Germany) for $2 \mathrm{~h}$ at $56^{\circ} \mathrm{C}$ and $2.5 \mathrm{~g} / \mu \mathrm{l}$ proteinase $\mathrm{K}$ (Roche) for $2 \mathrm{~h}$ at $37^{\circ} \mathrm{C}$, and only fragmented DNA was extracted. DNA was ethanol-precipitated and finally resuspended in distilled water. The fragmented DNA was electrophoretically fractionated on $1.5 \%$ agarose gel and stained with ethidium bromide.

\section{Nuclear condensation stained with DAPI}

H9c2 cells grown on $6 \mathrm{~mm}$ plate were washed twice with PBS and fixed for $30 \mathrm{~min}$ in $4 \%$ buffered paraformaldehyde. The cells were then stained with $1 \mu \mathrm{g} / \mathrm{ml} \mathrm{4}$,6-diamidine-2-phenylindole dihydrochloride (DAPI, Roche) for $30 \mathrm{~min}$. The stained cells were examined with fluorescence microscopy. The nuclei condensation was expressed by percentage (\%) of cell death (nuclei condensation) and expressed by relative percentage (\%) of $P$. gingivalis induced cell death (nuclei condensation).

\section{Western blot analysis}

Cultured H9c2 cells were scraped and washed once with phosphate-buffered saline. Cell suspension was then spun down, and cell pellets were lysed for $30 \mathrm{~min}$ in lysis buffer (50 mM Tris (pH 7.5), $0.5 \mathrm{M}$ $\mathrm{NaCl}, 1.0 \mathrm{mM}$ EDTA (pH 7.5), 10\% glycerol, $1 \mathrm{mM}$ BME, $1 \%$ IGEPAL-630 and proteinase inhibitor cocktail tablet (Roche)) and spun down $12,000 \mathrm{rpm}$ for $10 \mathrm{~min}$. Then, the supernatants were applied to new eppendorf tube for Western blot analysis. Proteins from $\mathrm{H} 9 \mathrm{c} 2$ cell line was then separated in $12 \%$ gradient SDS-PAGE and transferred to nitrocellulose membranes. Nonspecific protein binding was blocked in blocking buffer $(5 \%$ milk, $20 \mathrm{mM}$ Tris- $\mathrm{HCl}, \mathrm{pH} 7.6,150 \mathrm{mM}$ $\mathrm{NaCl}$, and $0.1 \%$ Tween 20) and blotted with specific antibodies of Bcl2-associated death promoter (Bad), and NFAT-3 (Santa Cruz Biotechnology, Santa Cruz, CA, USA), and $\alpha$-tubulin (Neo Markers, Fremont, CA, USA) as indicated for each experiment in the blocking buffer at $4{ }^{\circ} \mathrm{C}$ overnight.

\section{Experimental protocols}

\section{Protocol 1}

To quickly assess whether calcineurin signaling pathways were regulated by the periodontitis pathogen $P$. gingivalis in myocardial cell, calcineurin total protein and its direct downstream transcriptional effector, NFAT-3, and Bad proteins were measured by Western blotting in $\mathrm{H} 9 \mathrm{c} 2$ cells cultured in serum-free medium (control) for $4 \mathrm{~h}$ or serum-free medium plus 10 -fold diluted $(10 \times$ P. g) for $0.5 \mathrm{~h}$ (more details in Figure 1). 

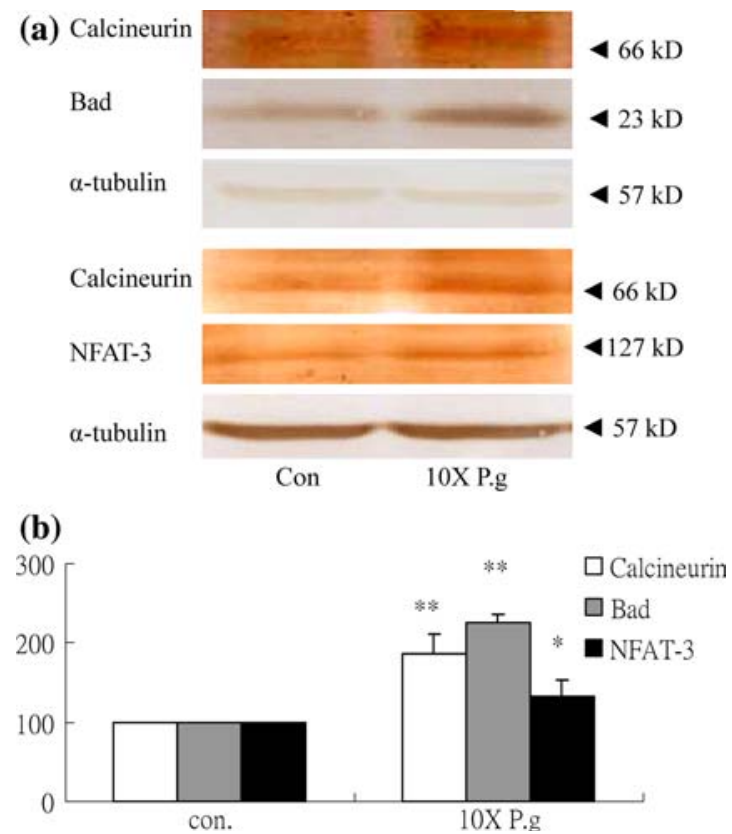

Figure 1. (a) The calcineurin, Bad, NFAT-3 protein products were measured by Western Blotting in $\mathrm{H} 9 \mathrm{c} 2$ cells cultured in serum-free medium (control) for $4 \mathrm{~h}$ and then treated with or without 10 -fold diluted Porphyromonas gingivalis $(10 \times \mathrm{P} . \mathrm{g})$ for $0.5 \mathrm{~h}$. An internal control of $\alpha$-tubulin was measured. (b) Bars represent the relative densitometry analysis of calcineu$\operatorname{rin} / \alpha$-tubulin, bad/ $\alpha$-tubulin and NFAT-3/ $\alpha$-tubulin to the control levels and indicate mean values \pm SD. $(n=6)$ ${ }^{*} p<0.05,{ }^{* *} p<0.01$, significant different between $P$. gingivalis and control.

\section{Protocol 2}

To assess whether calcineurin signaling pathways mediate $P$. gingivalis-induced myocardial cell hypertrophy, the cellular sizes were measured by actin-immunofluorescence in $\mathrm{H} 9 \mathrm{c} 2$ myocardial cells treated with either serum-free medium (control), or 10-fold diluted $P$. gingivalis for $24 \mathrm{~h}$ with or without calcineurin inhibitors. Beside $P$. gingivalis induced calcineurin and NFAT-3 in H9c2 cells were observed for $24 \mathrm{~h}$ (more details in Figure 2).

\section{Protocol 3}

To evaluate whether calcineurin is related to $P$. gingivalis-induced Bcl2-associated death promoter $(\mathrm{Bad})$, the protein products of calcineurin and Bad were measured by Western blotting analysis in myocardial $\mathrm{H} 9 \mathrm{c} 2$ cells with preadministration of serum-free medium (control) or calcineurin inhibitors (CsA) for $1 \mathrm{~h}$ and with postadministration of bacterial medium containing 10-fold diluted $P$. gingivalis medium for $0.5,1$, and $2 \mathrm{~h}$ (more details in Figure 3).

\section{Protocol 4}

To assess whether calcineurin signaling pathways mediate $P$. gingivalis induced myocardial cell apoptosis, DNA fragmentations were measured twice by agarose gel electrophoresis in $\mathrm{H} 9 \mathrm{c} 2$ myocardial cells with pre-administration of serum-free medium (control) or calcineurin inhibitors (CsA) for $1 \mathrm{~h}$ and with post-administration of bacterial medium containing 10-fold diluted $P$. gingivalis medium for $24 \mathrm{~h}$ (more details in Figure 4).

\section{Protocol 5}

To assess whether calcineurin signaling pathways mediate $P$. gingivalis induced myocardial cell apoptosis, nuclei condensation was measured by DAPI (4,6-diamidino-2-phenylindole) staining in myocardial H9c2 cells with pre-administration of serum-free medium (control) or calcineurin inhibitors (CsA) for $1 \mathrm{~h}$ and with post-administration of bacterial medium containing 10 -fold or 100 -fold diluted $P$. gingivalis medium for $24 \mathrm{~h}$ (more details in Figure 5).

\section{Statistics}

The nuclei condensation and cellular size of myocardial H9c2 cell were compared among groups treated with either control, 10-fold diluted $P$. gingivalis, and pre-treated calcineurin inhibitors using one-way analysis of variance (ANOVA) with pre-planned contrast comparison with control group and 10 -fold diluted $P$. gingivalis group. In all cases, a difference at $p<0.05$ was considered statistically significant. All data presented in the bar figures are means $\pm S D(n=6)$.

\section{Results}

Total calcineurin, activated Bad and NFAT-3 proteins were induced by $\mathrm{P}$. gingivalis

Western blot analysis revealed that total calcineurin, activated Bad and NFAT-3 proteins were gradually increased in $\mathrm{H} 9 \mathrm{c} 2$ cells after administration of 10 -fold diluted $P$. gingivalis $(10 \times \mathrm{P}$. g) for $0.5 \mathrm{~h}$. compared with cultured in serum-free medium (control) (Figure 1). 
(a)

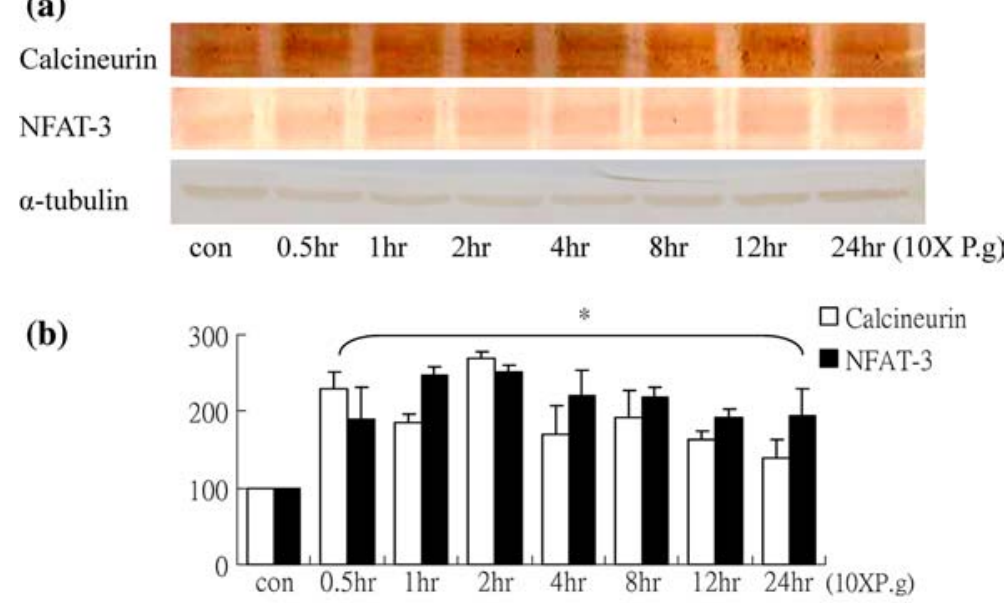

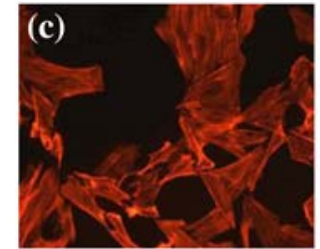

Con $(100 \%)$

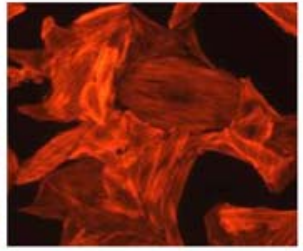

10X P.g

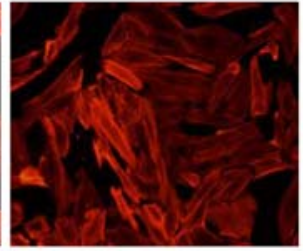

10X P.g + CsA

(d)

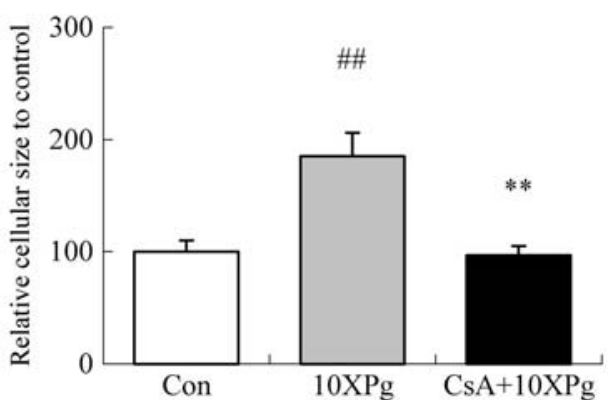

Figure 2. (a) Calcineurin and NFAT-3 protein products induced 10-fold diluted Porphyromonas gingivalis $(10 \times$ P. g) for 24 h. (b) Bars represent the relative densitometry analysis of calcineurin/ $\alpha$-tubulin and NFAT-3/ $\alpha$-tubulin to the control levels and indicate mean values \pm SD. $(n=6) * p<0.05$, significant different between $P$. gingivalis and control. (c) Cellular morphology was measured by actin-immunofluorescence in H9c2 myocardial cells treated with either control (Con, left panel), 10-fold diluted Porphyromonas gingivalis medium only $(10 \times \mathrm{P}$. g, middle panel), or pre-treated calcineurin inhibitors cyclosporin $\mathrm{A}(\mathrm{CsA}, 10 \mu \mathrm{M} / \mathrm{ml})$ for $1 \mathrm{~h}$, and then post-treated 10 -fold diluted $P$. gingivalis medium for $24 \mathrm{~h}(10 \times \mathrm{P} . \mathrm{g}+\mathrm{CsA}$, right panel) (d) Bars represent the relative cellular

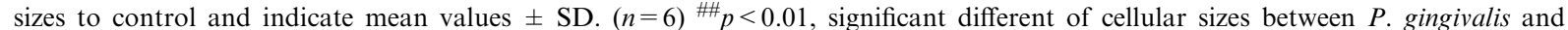
control, ${ }^{* *} p<0.01$, significant different of cellular areas between $P$. gingivalis and pre-treated signaling pathway inhibitors plus P. gingivalis.

Calcineurin mediate $\mathrm{P}$. gingivalis-induced myocardial cell hypertrophy

$P$. gingivalis-induced total calcineurin and NFAT-3 were not only shown in early $0.5 \mathrm{~h}$ but was also observed for 1, 2, 4, 8, 12, and $24 \mathrm{~h}$ (Figure $2 \mathrm{a}, 2 \mathrm{~b})$. Actin-immunofluorescence revealed that the increased cellular sizes by $85 \%$ were ob- served in myocardial $\mathrm{H} 9 \mathrm{c} 2$ cells treated with 10 fold diluted $P$. gingivalis medium for $24 \mathrm{~h}$ compared with serum-free control (Figure 2c). The $P$. gingivalis (10-fold diluted)-induced myocardial cell hypertrophy were significantly reduced by pre-administration of CsA (calcineurin inhibitor, $49 \%$ relative to cellular size of $10 \times$ P. g) (Figure 2d). 

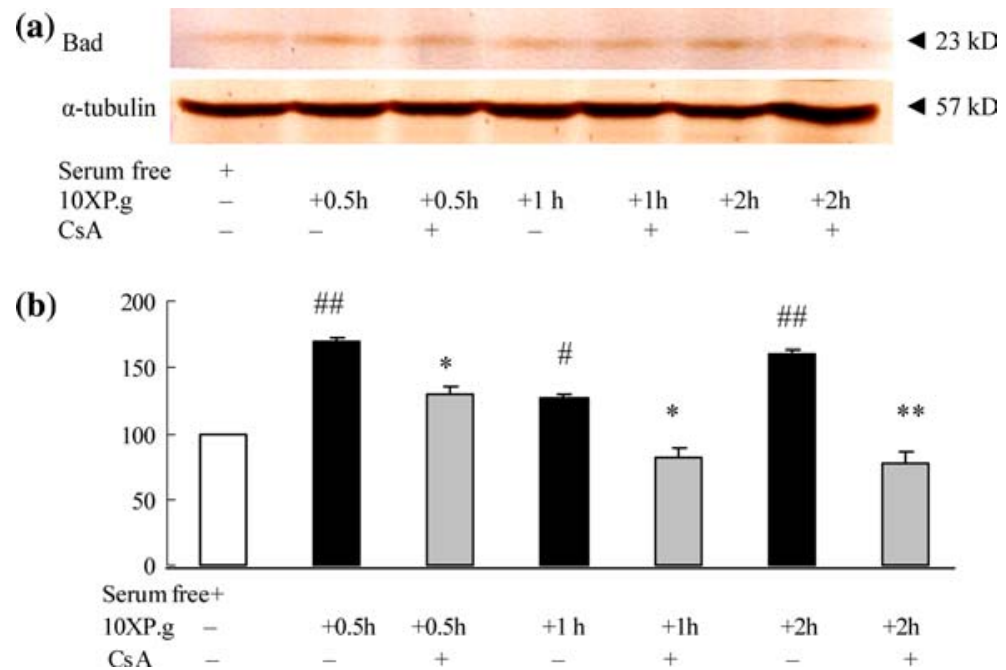

Figure 3. (a) The represented protein products of mitochondrial Bcl2-associated death promoter (Bad) were measured by Western Blotting in $\mathrm{H} 9 \mathrm{c} 2$ cells cultured in serum-free medium (control) for $4 \mathrm{~h}$ or treated with 10 -fold diluted $P$. gingivalis $(10 \times$ P. g) for $0.5 \mathrm{~h}, 1 \mathrm{~h}$, and $2 \mathrm{~h}$ with or without pre-administration of calcineurin inhibitors cyclosporin A (CsA, 0.5 $\mu \mathrm{M} / \mathrm{ml}$ ) before $1 \mathrm{~h}$. An internal control of $\alpha$-tubulin was measured. (b) Bars represent the relative densitometry analysis of $\mathrm{Bad} / \alpha$-tubulin to the control level and indicate mean values $\pm \mathrm{SD}$. $(n=6){ }^{\#} p<0.05{ }^{\# \#} p<0.01$, significant different between $P$. gingivalis and control, ${ }^{*} p<0.05,{ }^{* *} p<0.01$, significant different between $P$. gingivalis and pre-treated CsA plus P. gingivalis.

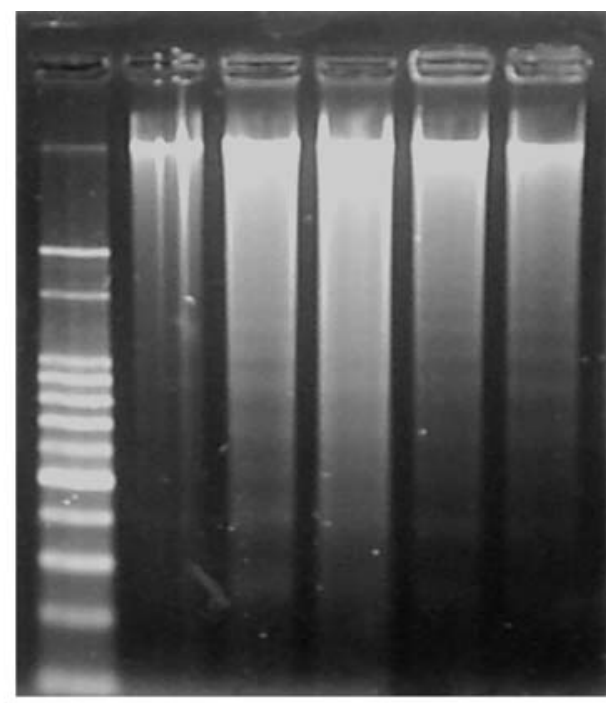

Marker $+$

Serum free

$\begin{array}{lllll}\text { 10xP.g } & + & + & + & + \\ \text { CsA } & & + & +\end{array}$

Figure 4. Represented DNA fragmentations were measured by agarose gel electrophoresis in H9c2 myocardial cells. H9c2 cells were treated with either serum free for $4 \mathrm{~h}$ (control), pretreated with or without calcineurin inhibitors cyclosporin $\mathrm{A}$ (CsA, $0.5 \mu \mathrm{M} / \mathrm{ml}$ ) for $1 \mathrm{~h}$, and then treated with bacterial medium containing 10-fold diluted Porphyromonas gingivalis $(10 \times$ P. g) for $24 \mathrm{~h}$.
Calcineurin mediate P. gingivalis induced Bad measured by Western blotting

Western blot analysis revealed that mitochondrial Bcl2-associated death promoter $(\mathrm{Bad})$ protein products were increased after administration of 10-fold diluted P. gingivalis $(10 \times \mathrm{P}$. g) for $0.5,1$, and $2 \mathrm{~h}$ (Figure 3 ). The increased activated Bad protein induced by 10 -fold diluted $P$. gingivalis in H9c2 cell were significantly reduced after preadministration of CsA (calcineurin inhibitor) (Figure 3).

Calcineurin mediate $\mathrm{P}$. gingivalis -induced myocardial cell apoptosis measured by DNA fragmentation

DNA ladder formation revealed that H9c2 myocardial cell underwent DNA fragmentation and apoptosis on exposure to either bacterial medium containing 10 -fold diluted $P$. gingivalis $(10 \times \mathrm{P}$. g) for $24 \mathrm{~h}$ compared to the serum free control (Figure 4). The 10-fold diluted $P$. gingivalis induced DNA fragmentation was attenuated by CsA (calcineurin inhibitor). 

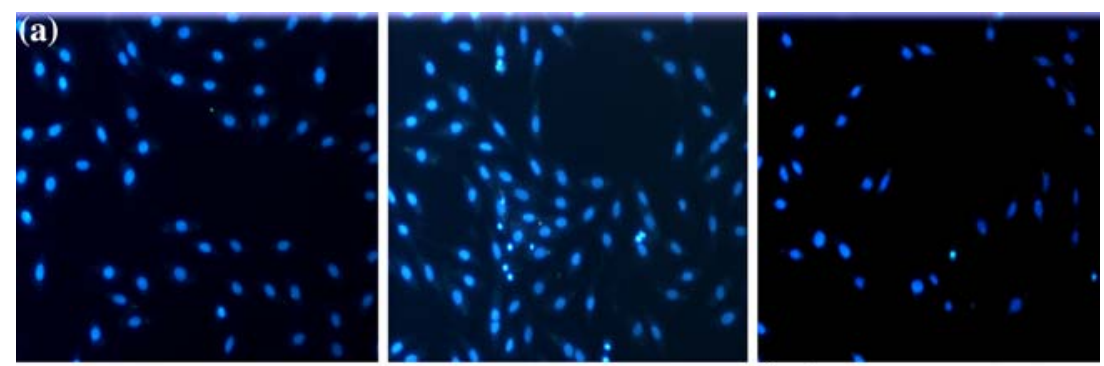

Con $(3.21 \%)$ 10X P.g (10.38\%)

CsA +10X P.g (7.29\%)
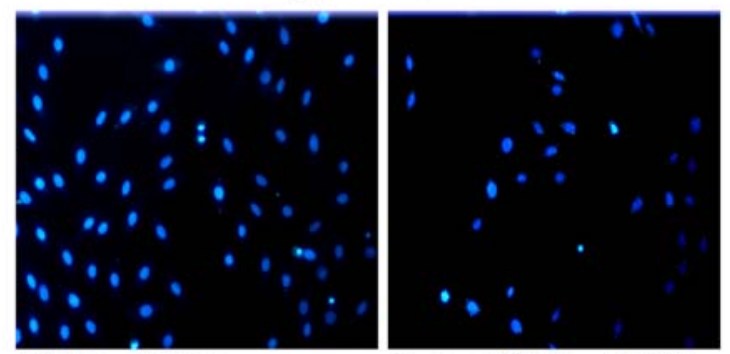

100X P.g $(6.69 \%)$

CsA +100X P.g (6.38\%)

(b)

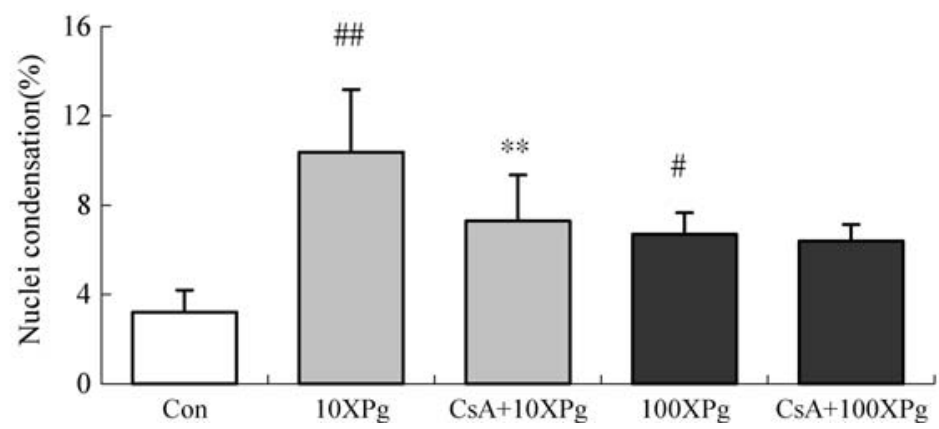

Figure 5. (a)Represented nuclei condensation expressed by \% of cell death (nuclei condensation) was measured by DAPI (4,6diamidino-2-phenylindole) $(1 \mu \mathrm{g} / \mathrm{ml})$ staining in H9c2 myocardial cells treated with either control (Con, upper left panel), 10-fold diluted Porphyromonas gingivalis medium (10X P. g, upper middle panel), pre-treated calcineurin inhibitors cyclosporin A (CsA; $0.5 \mu \mathrm{M} / \mathrm{ml}$ ) plus post-treated 10 -fold diluted bacterial medium $(\mathrm{CsA}+10 \times \mathrm{P}$. g, upper right panel), 100 -fold diluted $P$. gingivalis medium $(100 \times$ P. g, lower left panel), pre-treated CsA plus post-treated 100 -fold diluted $P$. gingivalis bacterial medium (CsA $+100 \times$ P. g, lower right panel) (b) Graphied by $\%$ of cell death. ${ }^{\#} p<0.05{ }^{\#} p<0.01$, significant different of $\%$ cell death (nuclei condensation) between $P$. gingivalis and control, $* * p<0.01$, significant different of $\%$ cell death (nuclei condensation) between $P$. gingivalis and pre-treated calcineurin inhibitors plus $P$. gingivalis.

Calcineurin mediate $\mathrm{P}$. gingivalis-induced myocardial cell apoptosis measured by condensed nuclei with DAPI staining

The condensation of nuclei stained with DAPI, indicating cell apoptosis, was significantly increased after administration of 10 -fold diluted $P$. gingivalis for $24 \mathrm{~h}(10 \times \mathrm{P} . \mathrm{g}, p<0.01)$ and 100 -fold diluted $P$. gingivalis for $24 \mathrm{~h}(100 \times \mathrm{P} . \mathrm{g}, p<0.05)$ compared with control (Con) (Figure 5). The 100-fold diluted $P$. gingivalis-induced condensed nuclei in $\mathrm{H} 9 \mathrm{c} 2$ cell show no differences between with or without CsA (calcineurin inhibitor), whereas the increased condensed nuclei induced by 10 -fold diluted $P$. gingivalis were significantly $(p<0.01)$ reduced after pre-administration of CsA.

\section{Discussion}

Major findings

Our main findings can be summarized as follows: (1) Total calcineurin, activated Bad and NFAT-3 protein in $\mathrm{H} 9 \mathrm{c} 2$ cells were upregulated by $P$. gingivalis. (2) $P$. gingivalis increased $\mathrm{H} 9 \mathrm{c} 2$ cellular sizes, DNA 
fragmentation, and nuclear condensation in $\mathrm{H} 9 \mathrm{c} 2$ myocardial cells, which imply $P$. gingivalis induced myocardial cellular hypertrophy and apoptosis. (3)The increases in cellular sizes, DNA fragmentation, nuclear condensation, and pro-apoptoric Bad protein of $\mathrm{H} 9 \mathrm{c} 2$ cells treated with $P$. gingivalis were all reduced after pre-administration of CsA. In sum, $P$. gingivalis-induced myocardial hypertrophy and apoptosis appear to be mediated by calcineurin/ NFAT or calcineurin/Bad signaling pathway. These findings provide a potential link between $P$. gingivalis and calcineurin activity. After integrating previous theories and our current findings into one, we hypothesize that that myocardial cell hypertrophy and myocardial cell apoptosis will be induced by $P$. gingivalis via calcineurin/NFAT-3 pathway and calcineurin/Bad pathway, respectively (shown in Figure 6).

\section{Experimental limitation}

P. gingivalis, a gram-negative anaerobe, is involved in the pathogenesis of periodontal disease and plays important roles in eliciting cellular responses in various ways [18]. $P$. gingivalis possesses a variety of virulence factors including gingipains, fimbrillin peptides, capsule polysaccharides, lipopolysaccharides, hemagglutinating and hemolysing activities, toxic products of metabolism, outer membrane vesicles, and other enzymes [19]. Because of the widespread virulence factors, $P$. gingivalis can produce myocardial cell apoptosis via various virulence factors or metabolic byproducts. Therefore, we have to add a note of caution that any effect noted in the present investigation

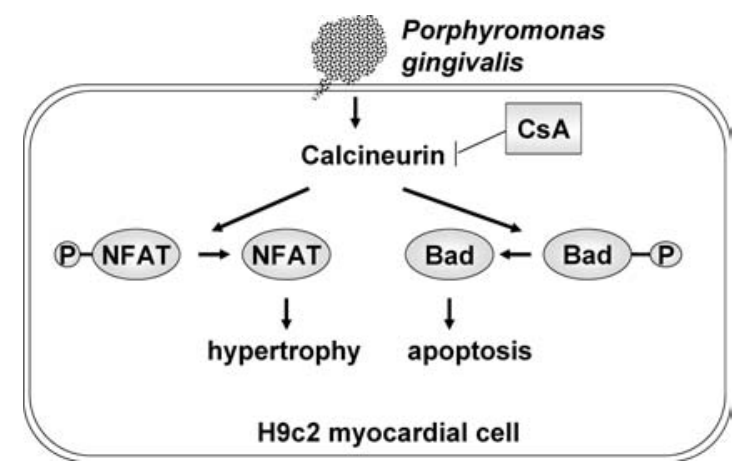

Figure 6. After integrating previous theories and our current findings into one, we hypothesized that myocardial cell hypertrophy and myocardial cell apoptosis may be induced by porphyromonas gingivalis via calcineurin/NFAT-3 pathway and calcineurin/Bad pathway, respectively. cannot be isolated to any specific virulence factor or any metabolic byproduct. Nevertheless, the goal of the present study was to clarify the role of calcineurin signaling pathway in $P$. gingivalisinduced myocardial cell apoptosis. Clearly, additional experiments using a reductionist approach, such as investigating gingipains alone or lipopolysaccharides alone, will be required in order to identify which specific virulence factors or metabolic byproducts directly cause myocardial cell apoptosis.

\section{P. gingivalis and cardiovascular system}

Some studies indicated that $P$. gingivalis did affect the cardiovascular system, such as the coronary arteries $[3,20]$. P. gingivalis was suggested to involve in atherosclerotic lesion formation [20] and was found in the aorta of some cardiovascular patients who had undergone open-heart surgery [21], the latter of which suggests that periodontal pathogen $P$. gingivalis did invade the cardiovascular system and might involve cardiovascular diseases [21]. Most cross-sectional, prospective epidemiological studies, animal model studies, and cellular studies have demonstrated that periodontal disease ( $P$. gingivalis) may be associated with endothelial cell, atherosclerosis formation, and coronary heart disease $[1-3,6,20,21]$. Myocardial ischemia and infarction are often preceded by acute thromboembolic events induced by periodontal disease, such as $P$. gingivalis [2]. In our previous studies, $P$. gingivalis increased apoptosis and the activated apoptotic caspase-3, caspase- 8 , and caspase- 9 proteins of cardiomyocyte $\mathrm{H} 9 \mathrm{c} 2$ cells as well as $P$. gingivalis-induced apoptotic activity of $\mathrm{H} 9 \mathrm{c} 2$ cells were involved in $\mathrm{p} 38$, ERK, PI3K, and calcineurin pathway, but might play a protective role via JNK pathway [5]. In the current study, we observed the two major calcineurin-mediated pathways in $P$. gingivalis-induced myocardial cell hypertrophy and apoptosis.

\section{$\mathrm{P}$. gingivalis and calcineurin}

Calcineurin, a $\mathrm{Ca}^{2+}$-calmodulin-dependent protein phosphatase is one of the links between $\mathrm{Ca}^{2+}$ signals and regulation of gene transcription in cardiac muscle. Calcineurin has been implicated in cardiovascular disease and is activated by numerous stimuli, such as hypertension, ischemic/ 
re-perfusion, hypoxia, pressure-overload stress, increase in cardiac pacing frequency [14, 22-24] and periodontitis pathogens $P$. gingivalis found in the current study. The current findings indicated that $P$. gingivalis-induced $\mathrm{H} 9 \mathrm{c} 2$ cell hypertrophy and apoptosis was mediated by calcineurin signaling pathways. Herein, our findings imply that calcineurin signaling pathway in myocardial cell can be activated by one more cellular stimulus, $P$. gingivalis, i.e. periodontal infection. To our knowledge, no other study looking into the direct or indirect interaction between $P$. gingivalis and calcineurin activity in all types of cells or systems. This finding might provide a potential link between $P$. gingivalis and calcineurin signaling pathway. Therefore, more studies in the interaction between $P$. gingivalis and calcineurin signaling pathway in cardiovascular systems are needed.

\section{Role of calcineurin in $\mathrm{P}$. gingivalis-induced myocardial cell hypertrophy}

The heart is a dynamic organ capable of adapting its size and architecture in response to various stressors or bacterial infection [25, 26]. Our findings showed that myocardial cell sizes, total calcineurin, and activated NFAT-3 were all increased in $\mathrm{H} 9 \mathrm{c} 2$ cells by 10 -fold diluted $P$. gingivalis challenge and the increased myocardial cell sizes-induced by $P$. gingivalis were reduced by calcineurin inhibitor, suggesting that myocardial cell hypertrophy will induced by $P$. gingivalis via calcineurin/NFAT-3 pathway. The current findings provide a potential link between $P$. gingivalis and calcineurin/NFAT-3 or calcineurin/Bad pathway in myocardial cell hypertrophy or apoptosis. Calcineurin/NFAT was shown to induce up-regulation of the Fas death apoptotic pathway in neuronal cells [27]. Therefore, there is a limitation in our study to clarify the relative role of NFAT or Bad in hypertrophy or apoptosis. Further studies are required to confirm the role of $P$. gingivalis on cardiac hypertrophy or heart failure via calcineurin/NFAT-3 pathway.

Calcineurin role in $\mathrm{P}$. gingivalis-induced myocardial cell apoptosis

Apoptosis is characterized by DNA-fragmentation, nuclear condensation, and shifting the balance between anti-apoptotic and pro-apoptotic proteins towards the latter $[14,15]$. The mitochondrial Bcl2 family plays an important role in cardiac apoptosis. Shifting the balance between anti-apoptotic and pro-apoptotic Bcl2 family proteins towards the latter (e.g. increased Bad proteins) will cause releasing cytochrome $c$ into cytosol and activate caspase 9, which will activate caspase 3 that is responsible for cleavage action and DNA fragmentation. Our findings showed that periodontal pathogen $P$. gingivalis increased DNA fragmentation, nuclear condensation, and activated Bad protein in $\mathrm{H} 9 \mathrm{c} 2$ cells. All increases were attenuated by calcineurin inhibitor, which suggest that $P$. gingivalis induce $\mathrm{H} 9 \mathrm{c} 2$ myocardial cell apoptosis via calcineurin signaling pathway and $P$. gingivalis-induced myocardial cell apoptosis may be involve in Bad related apoptotic pathways.

Calcineurin plays a critical role in the proapoptosis of cardiac myocytes through dephosphorylating pro-apoptotic protein Bad to promote apoptosis. Our findings showed that the increased Bad proteins induced by $0.5-2 \mathrm{~h} P$. gingivalis challenges were attenuated by calcineurin inhibitors, which can be explained by the supposition that calcineurin inhibitors may inhibit dephosphorylation of Bad and decrease activated Bad protein level. Therefore, we can generally conclude that calcineurin activity may be initiated by $P$. gingivalis and may further activate Bad related apoptotic pathways to lead cell death in culture H9c2 myocardial cells. However, 100-fold diluted $\mathrm{P}$. gingivalis induced myocardial cell nuclear condensation were not inhibited by CsA in the current study. Therefore, we have to make a cautious note that more diluted $P$. gingivalisinduced myocardial cell apoptosis may not be mediated via calcineurin signaling pathway.

\section{Further hypotheses for clinical application}

Our findings can provide a future hypothesis that myocardial cell hypertrophy and apoptosis may potentially gradually occur in patients with periodontitis infected by $P$. gingivalis, which might be mediated by calcineurin signaling pathways. In order to prevent potential cardiac diseases, such as pathologic cardiac hypertrophy or heart failure, in periodontitis patients infected by $P$. gingivalis, the infected patients have been suggested that they should ideally maintain excellent oral hygiene 
and suppress $P$. gingivalis infection as much as possible, and our findings further proposed that calcineurin signaling pathways inhibitors may be a good candidate for developing a future therapeutic approach to prevent $P$. gingivalis-induced cardiac hypertrophy and/or heart failure. Of course, further clinical studies are needed to prove or disprove our hypotheses for clinical application.

\section{References}

1. Genco R., Offenbacher S. and Beck J., Periodontal disease and cardiovascular disease: epidemiology and possible mechanisms. J. Am. Dent. Assoc. 133(Suppl): 14S-22S, 2002.

2. Herzberg M.C. and Weyer M.W., Dental plaque, platelets, and cardiovascular diseases. Ann. Periodontol. 3: 151-160, 1998.

3. Kuramitsu H.K., Qi M., Kang I.C. and Chen W., Role for periodontal bacteria in cardiovascular diseases. Ann. Periodontol. 6: 41-47, 2001.

4. Fortuno M.A., Lopez N., Gonzalez A. and Diez J., Involvement of cardiomyocyte survival-apoptosis balance in hypertensive cardiac remodeling. Expert Rev. Cardiovasc. Ther. 1: 293-307, 2003.

5. Lee S.D., Wu C.C., Kuo W.W., Lin J.A., Huang C.M., Lu M.C., Chen L.M., Hsu H.H., Wang C.K. and Chang S.H., Porphyromonas gingivalis-related cardiac cell apoptosis was majorly co-activated by p38 and ERK pathways. J. Periodontal Res. 2006 (in press).

6. Deshpande R.G., Khan M.B. and Genco C.A., Invasion of aortic and heart endothelial cells by Porphyromonas gingivalis. Infect. Immun. 66: 5337-5343, 1998.

7. Takano H., Zou Y., Akazawa H., Toko H., Mizukami M., Hasegawa H., Asakawa M., Nagai T. and Komuro I., Inhibitory molecules in signal transduction pathways of cardiac hypertrophy. Hypertens. Res. 25: 491-498, 2002.

8. Narula J., Pandey P., Arbustini E., Haider N., Narula N., Kolodgie F.D., Dal Bello B., Semigran M.J., BielsaMasdeu A., Dec G.W., Israels S., Ballester M., Virmani R., Saxena S. and Kharbanda S., Apoptosis in heart failure: release of cytochrome $\mathrm{c}$ from mitochondria and activation of caspase-3 in human cardiomyopathy. Proc. Natl. Acad. Sci. USA 96: 8144-8149, 1999.

9. Gill C., Mestril R. and Samali A., Losing heart: the role of apoptosis in heart disease-a novel therapeutic target? Faseb J. 16: 135-146, 2002.

10. Dhalla N.S., Ziegelhoffer A., Singal P.K., Panagia V. and Dhillon K.S., Subcellular changes during cardiac hypertrophy and heart failure due to bacterial endocarditis. Basic Res. Cardiol. 75: 81-91, 1980.

11. Saraste A., Morphologic criteria and detection of apoptosis. Herz 24: 189-195, 1999.

12. Bishopric N.H., Andreka P., Slepak T. and Webster K.A., Molecular mechanisms of apoptosis in the cardiac myocyte. Curr. Opin. Pharmacol. 1: 141-150, 2001.

13. Schulz R.A. and Yutzey K.E., Calcineurin signaling and NFAT activation in cardiovascular and skeletal muscle development. Dev. Biol. 266: 1-16, 2004.
14. Saito S., Hiroi Y., Zou Y., Aikawa R., Toko H., Shibasaki F., Yazaki Y., Nagai R. and Komuro I., beta-Adrenergic pathway induces apoptosis through calcineurin activation in cardiac myocytes. J. Biol. Chem. 275: 34528-34533, 2000 .

15. Wang H.G., Pathan N., Ethell I.M., Krajewski S., Yamaguchi Y., Shibasaki F., McKeon F., Bobo T., Franke T.F. and Reed J.C., $\mathrm{Ca}^{2+}$-induced apoptosis through calcineurin dephosphorylation of BAD. Science 284: 339343, 1999.

16. Wilkins B.J., Dai Y.S., Bueno O.F., Parsons S.A., Xu J., Plank D.M., Jones F., Kimball T.R. and Molkentin J.D., Calcineurin/NFAT coupling participates in pathological, but not physiological, cardiac hypertrophy. Circ. Res. 94: 110-118, 2004.

17. Diedrichs H., Chi M., Boelck B., Mehlhorn U. and Schwinger R.H., Increased regulatory activity of the calcineurin/NFAT pathway in human heart failure. Eur. J. Heart Fail. 6: 3-9, 2004.

18. Curtis M.A., Kuramitsu H.K., Lantz M., Macrina F.L., Nakayama K., Potempa J., Reynolds E.C. and AduseOpoku J., Molecular genetics and nomenclature of proteases of Porphyromonas gingivalis. J. Periodontal Res. 34: 464-472, 1999.

19. Kantarci A. and Van Dyke T.E., Neutrophil-mediated host response to Porphyromonas gingivalis. J. Int. Acad. Periodontol. 4: 119-125, 2002.

20. Kuramitsu H.K., Kang I.C. and Qi M., Interactions of Porphyromonas gingivalis with host cells: implications for cardiovascular diseases. J. Periodontol. 74: 85-89, 2003.

21. Stelzel M., Conrads G., Pankuweit S., Maisch B., Vogt S., Moosdorf R. and Flores-de-Jacoby L., Detection of Porphyromonas gingivalis DNA in aortic tissue by PCR. J. Periodontol. 73: 868-870, 2002.

22. Nagata K., Somura F., Obata K., Odashima M., Izawa H., Ichihara S., Nagasaka T., Iwase M., Yamada Y., Nakashima N. and Yokota M., AT1 receptor blockade reduces cardiac calcineurin activity in hypertensive rats. Hypertension 40: 168-174, 2002.

23. Lakshmikuttyamma A., Selvakumar P., Kakkar R., Kanthan R., Wang R. and Sharma R.K., Activation of calcineurin expression in ischemia-reperfused rat heart and in human ischemic myocardium. J. Cell. Biochem. 90: 987997, 2003.

24. Tavi P., Pikkarainen S., Ronkainen J., Niemela P., Ilves M., Weckstrom M., Vuolteenaho O., Bruton J., Westerblad H. and Ruskoaho H., Pacing-induced calcineurin activation controls cardiac $\mathrm{Ca} 2+$ signalling and gene expression. J. Physiol. 554: 309-320, 2004.

25. Tomlinson C.W. and Dhalla N.S., Alteration in calcium metabolism in cardiac hypertrophy and failure caused by bacterial infection. Recent Adv. Stud. Cardiac Struct. Metab. 12: 191-198, 1976.

26. Ravingerova T., Barancik M. and Strniskova M., Mitogenactivated protein kinases: a new therapeutic target in cardiac pathology. Mol. Cell Biochem. 247: 127-138, 2003.

27. Jayanthi S., Deng X., Ladenheim B., McCoy M.T., Cluster A., Cai N.S. and Cadet J.L., Calcineurin/NFAT-induced up-regulation of the Fas ligand/Fas death pathway is involved in methamphetamine-induced neuronal apoptosis. Proc. Natl. Acad. Sci. USA 102: 868-873, 2005. 